\title{
Esteatose hepática aguda da gravidez: relato de caso e revisão de literatura
}

\section{Acute fatty liver of pregnancy: case report and literature review}

Rômulo Pedroza Pinheiro ${ }^{1}$, Ivelise Regina Canito Brasil ${ }^{2}$, Shirley Kelly Bede Bruno ${ }^{3}$, Ticiana Mota Esmeraldo ${ }^{4}$, Marina Madeira Castelo Branco ${ }^{5}$, Ricardo Bezerra Walraven ${ }^{1}$

\begin{abstract}
RESUMO
A esteatose hepática aguda da gravidez (EHAG) é uma complicação obstétrica rara e grave, que ocorre durante o terceiro trimestre de gestação. O objetivo deste estudo é o relato de caso de EHAG em paciente internada em hospital terciário. Gestante, 36 anos, na $37^{a}$ semana de gravidez previamente hígida até quatro dias antes da admissão hospitalar. Iniciou quadro de dor abdominal em epigástrio e hipocôndrio direito, icterícia progressiva, desidratação, febre e calafrios. Evoluiu com piora dos sintomas, hematêmese, hipotensão e hipoglicemia. A função hepática apresentava alterações. O coagulograma revelava tempo de protrombina e de tromboplastina parcial alargados. Os marcadores virais para hepatites A, B e C foram negativos. No pós-operatório imediato da Cesárea, a paciente evoluiu com distúrbio da coagulação, episódios de hipoglicemia, piora da icterícia e encefalopatia hepática, caracterizando o diagnóstico de insuficiência hepática aguda (IHA). Após quatro dias de tratamento da encefalopatia hepática houve melhora do sensório até apresentar-se consciente, orientada, sem flapping, apenas com tratamento conservador e não preencheu os critérios do King's Collegue de indicação para transplante hepático. No $15^{\circ}$ dia de internação tanto a paciente e filho encontravam-se bem. Com o conhecimento e diagnóstico de EHAG, no reconhecimento precoce de casos mais leves, incluindo interrupção precoce da gravidez por cesariana e grandes volume de plasma fresco congelado e albumina, alternadamente, o prognóstico de EHAG pode melhorar.
\end{abstract}

Palavras-chave: Gravidez. Esteatose Hepática. Complicações na Gravidez. Insuficiência Hepática.

1. Graduando em Medicina da Universidade Estadual do Ceará UECE.

2. Professora do Curso de Graduação em Medicina da UECE e Cirurgiã Digestiva especialista em Transplante Hepático do Hospital Geral de Fortaleza - HGF.

3. Médica Preceptora Ginecologista e Obstetra do Hospital Geral de Fortaleza - HGF.

4. Médica Hepatologista do Hospital Geral de Fortaleza - HGF.

5. Graduanda em Medicina pela Universidade de Fortaleza UNIFOR.
Correspondência Hospital Geral de Fortaleza Rua. Ávila Goulart, 900 - Papicu CEP: 60.175-295 - Fortaleza, Ceará - Brasil romulopedroza@gmail.com

Artigo recebido em 30/08/2013 Aprovado para publicação em 10/10/2014

Instituição onde o trabalho foi desenvolvido: Hospital Geral de Fortaleza. 


\begin{abstract}
Acute fatty liver of the pregnancy (AFLP) is a rare but serious obstetric complication that occurs during the three last months of pregnancy. Our objective was to describe a AFLP case report of a patient from a tertiary hospital. A 36 years old on the $37^{\text {th }}$ week of pregnancy with a history of being previously healthy until the $4^{\text {th }}$ day of admission when she began a clinical picture of abdominal pain in epigastrium and right hypochondrium, progressive jaundice, dehydration, fever and chills. The patient evolved with worsening of symptoms with hematemesis, hypotension and hypoglycemia. Liver function was abnormal. The coagulation tests revealed a prothrombin time and partial thromboplastin extended. The markers for hepatitis viruses $A, B$ and $C$ were negative. In the immediate postoperative period of Caesarea, the patient with coagulation, hypoglycemia, worsening jaundice and hepatic encephalopathy disorder, featuring the diagnosis of acute liver failure (ALF). After 4 days of hepatic encephalopathy there was an improvement of her general state until she got conscient, oriented, without flapping, only with conservative tratamento and not fulfilling the criteria of King's College indication for liver transplantation. On the 15th day after admission the patient as much as his son were in good condition. With increasing knowledge of the diagnosis of AFLP, especially in the early recognition of milder cases, including early termination of pregnancy by caesarean section and large dose infusion of fresh frozen plasma and albumin, alternately, the prognosis may be better on AFLP cases.
\end{abstract}

Keywords: Pregnancy. Fatty Liver. Pregnancy Complications. Hepatic Insufficiency.

\section{Introduçåo}

Esteatose hepática aguda da gravidez (EHAG) é uma complicação obstétrica rara e grave, foi descrita pela primeira vez em 1940 por Sheehan como "acute yellow atrophy of the liver". ${ }^{1}$ A EHAG é caracterizada por infiltração de gordura no sistema microvesicular dos hepatócitos sem qualquer inflamação ou necrose. Tem incidência estimada de um caso para 10.000 a 15.000 partos, e ocorre comumente durante o terceiro trimestre de gestação. A mortalidade atribuída à EHAG é elevada, com taxas de $70 \%$ e $80 \%$ para feto e mãe, respectivamente. ${ }^{2-5}$ Entidade mais frequente em primíparas, porém pode ocorrer em multíparas com gestações prévias sem intercorrências. Pesquisas com biologia molecular sugerem que a EHAG pode ser o resultado da disfunção mitocondrial por deficiência da terceira enzima de cadeia longa da 3-Hidroxiacil CoA Desidrogenases. Além disso, outras características clínicas da gravidez podem ser consideradas fatores de risco em potencial: primigestas (primeira gestação), pré-eclâmpsia, feto do sexo masculino e gestação múltipla. ${ }^{6,7,8}$

Acomete a gestante entre as $28^{\mathrm{a}}$ e $40^{\mathrm{a}}$ semanas de gravidez com manifestações clínicas inespecíficas como náuseas, vômitos e dor abdominal, seguidos de icterícia após a primeira semana do início do quadro, dificultando o diagnóstico precoce. Em até $50 \%$ dos casos, algumas pacientes apresentam hipertensão arterial, edema periférico, seguidos de proteinúria, que se associando a síndrome HELLP e pré-eclâmpsia. ${ }^{9}$ A identificação tardia desta síndrome pode ocasionar forma grave de insuficiência hepática aguda (IHA) e encefalopatia com risco de vida por disfunção de múltiplos órgãos, em necessidade de se encaminhar a parturiente para centros terciários de suporte intensivo que contam com especialistas em gastrohepatologia para garantir o sucesso do tratamento materno e fetal. ${ }^{10} \mathrm{O}$ espectro das complicações hepáticas da EHAG é bastante variado, desde formas oligo ou assintomática até a IHA grave, essa, com elevada mortalidade sendo o transplante hepático considerado o único recurso terapêutico para garantir a sobrevida materna, geralmente indicado após 48 horas do parto quando não ocorre regeneração hepática. ${ }^{10-13}$

Por ser a EHAG de alta morbimortalidade, com necessidade imperiosa do diagnóstico precoce e interrupção da gravidez o que define o prognóstico da gestante e do bebê, o presente estudo tem o objetivo de relatar um caso de EHAG, com suas características clínicas, laboratoriais e condutas terapêuticas adotadas.

\section{Descrição do caso}

Gestante, 36 anos, na $37^{\mathrm{a}}$ semana de gravidez, primípara, parda, brasileira foi admitida em um hospital particular de Fortaleza, Ceará, com história de ser previamente hígida até quatro dias da admissão hospitalar. Iniciou quadro clínico de dor abdominal em epigástrio e hipocôndrio direito, icterícia progressiva, desidratação, febre e calafrios, evoluindo com piora dos sintomas, hematêmese, hipotensão e hipoglicemia. Avaliada pelo cirurgião geral com hipótese 
diagnóstica de colangite. Iniciado antibioticoterapia com ampicilina-sulbactam, e solicitado avaliação obstétrica que fez diagnóstico de EHAG, indicou a interrupção da gravidez.

No dia anterior da cesariana os resultados de laboratório mostraram contagem de plaquetas normais de $160.000 \mu \mathrm{L}$, glóbulos brancos de 15.000 e nível de glicose de $49 \mathrm{mg} / \mathrm{dL}$. A função hepática estava com elevação nos níveis séricos de bilirrubina total (10,55 $\mathrm{mg} / \mathrm{dL}$ ), transaminase pirúvica (TGP; $251 \mathrm{UI} / \mathrm{L}$ ), transaminase oxalacética (TGO: 277 UI / L) e fosfatase alcalina (FA: $642 \mathrm{U} / \mathrm{L}$ ). O coagulograma revelou um tempo de protrombina de 23,8 segundos e um tempo de tromboplastina parcial de 50,3 segundos. Os marcadores virais das hepatites A, B e C foram negativos, ultrassonografia abdominal total mostrou fígado de dimensões normais, contornos regulares, ecotextura aumentada difusamente, ausência de nódulos sólidos e/ou císticos e sem sinais de dilatações das vias biliares intra ou extra-hepáticas, não visualizando a vesícula biliar.

O tratamento de suporte foi instituido, incluindo fluidoterapia, nutrição enteral, fármacos vasoativos e correção da hipoglicemia, além de hemoderivados como plasma fresco congelado na tentativa de manter a razão normalizada internacional do tempo de protrombina (INR < 1,5) (Gráfico 1).
No pós-operatório a paciente evoluiu com distúrbio da coagulação (INR: 2,59), manteve-se hipoglicêmica $(65 \mathrm{mg} / \mathrm{dL})$, piora da icterícia e encefalopatia hepática, configurando o diagnóstico de insuficiência hepática aguda (IHA). Já em ambiente de terapia intensiva evoluiu com oligúria, mas não dialítica. Após quatro dias de encefalopatia hepática houve melhora do sensório até apresentar-se consciente, orientada, sem flapping, apenas com tratamento conservador e não preencheu os critérios do King's Collegue de indicação de transplante hepático.

A curva de evolução das bilirrubinas manteve-se no intervalo 9,29-19,82 mg/dL (Gráfico 2).

No $8^{\circ}$ dia após o parto, a hemoglobina atingiu o nível de $6,7 \mathrm{mg} / \mathrm{dL}$, com presença de volumoso hematoma de parede abdominal associado a ascite (vide Fig. 1) e ureterohidronefrose à direita (vide Fig. 2), e aumento da musculatura da parede abdominal no andar inferior do abdômen que se encontra heterogênea visto em ultrassonografia de parede abdominal, configurando uma complicação no pós-operatório com extenso hematoma subaponeurótico de parede abdominal com necessidade de abordagem cirúrgica e drenagem.

A função renal foi efetivamente restabelecida, com um aumento do volume urinário e redução da ansarca. No $15^{\circ}$ dia de internação tanto a paciente como seu filho estavam bem.

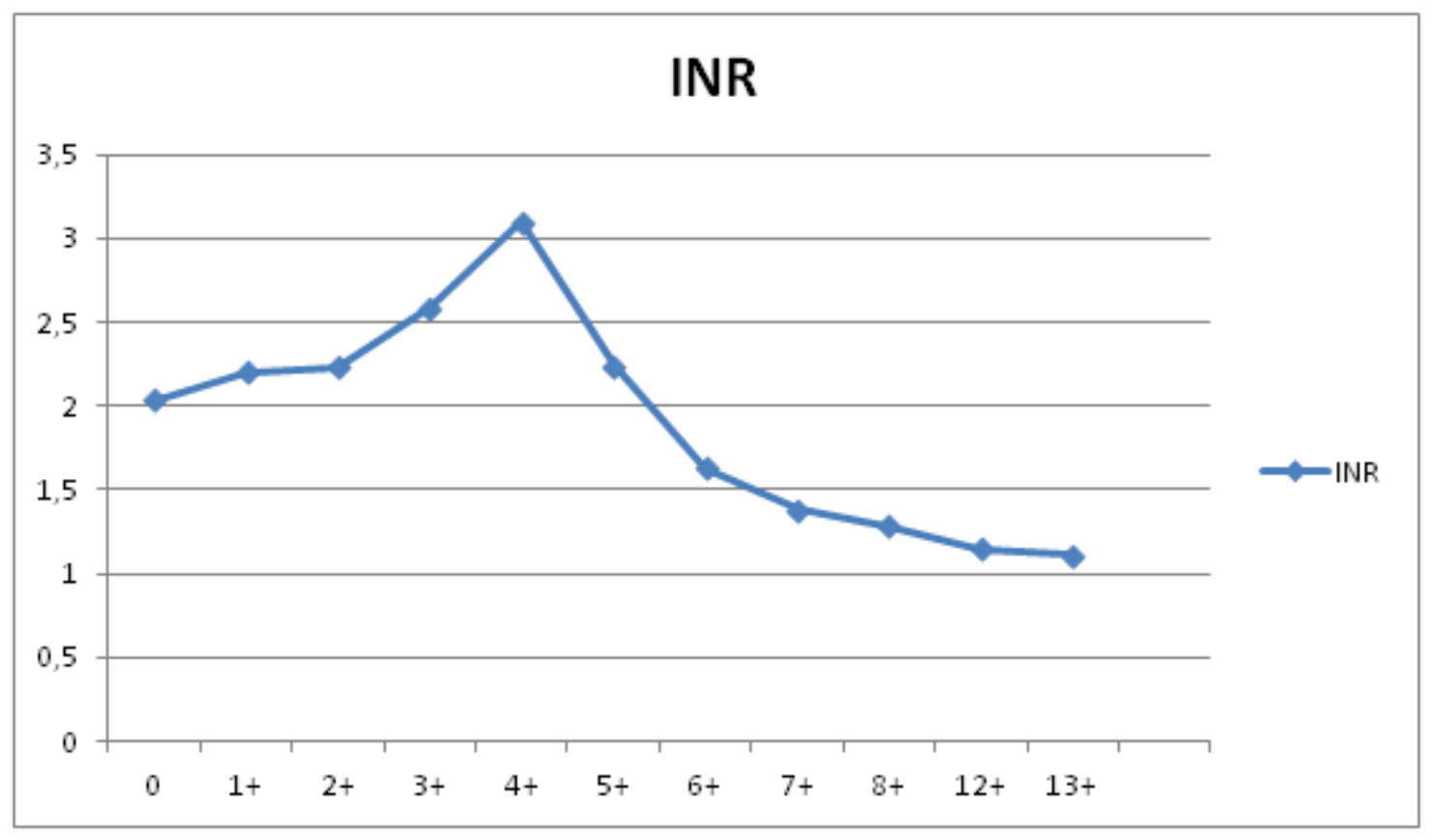

Gráfico 1 - Níveis de INR em função do tempo de parto. Valores positivos são pós-parto, considerando "0" o dia do parto. 


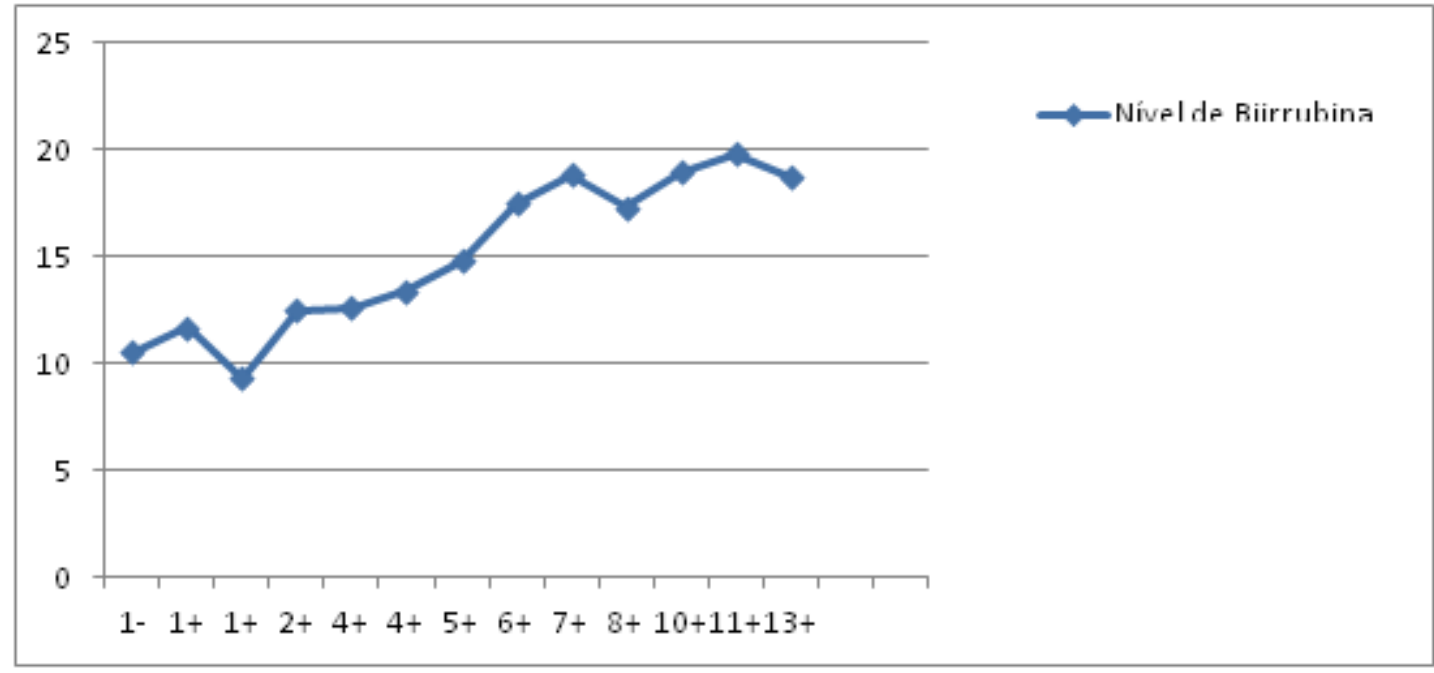

Gráfico 2 - Concentração de bilirrubina total, em mg/dL, em função do tempo de parto.

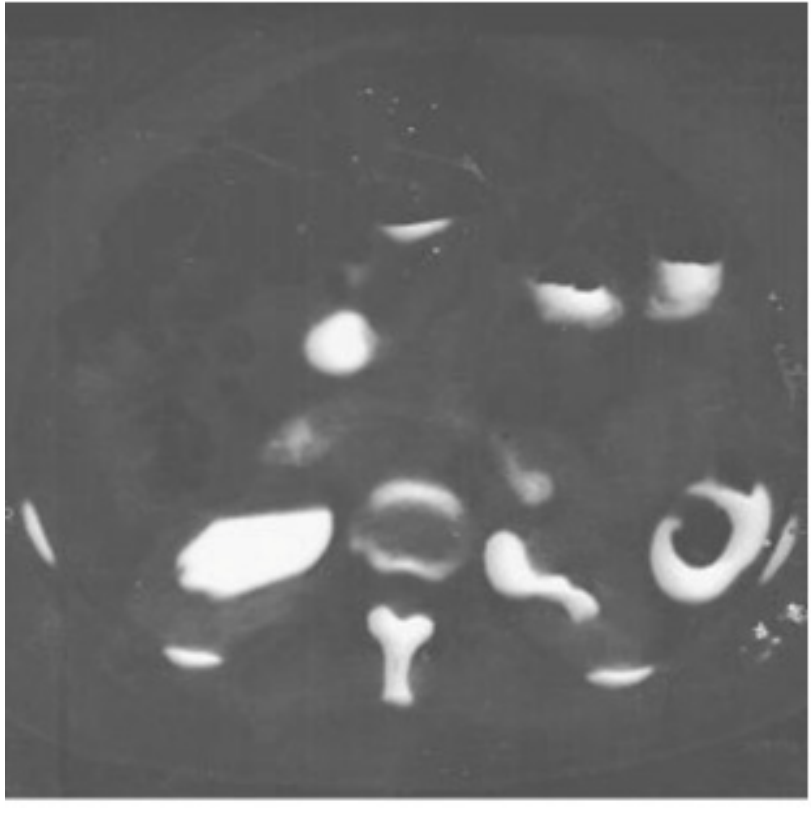

Figura 1 - TC de Abdome Superior (técnica multislice).

\section{Discussão}

Manifestações de pré-eclâmpsia são geralmente observados na segunda metade da gravidez, enquanto que os sintomas da síndrome HELLP e EHAG frequentemente aparecem no terceiro trimestre. A hepatite viral aguda da gravidez apresenta como uma doença sistêmica, com febre, náuseas, vômitos, fadiga e icterícia e níveis séricos de aminotransferases muito

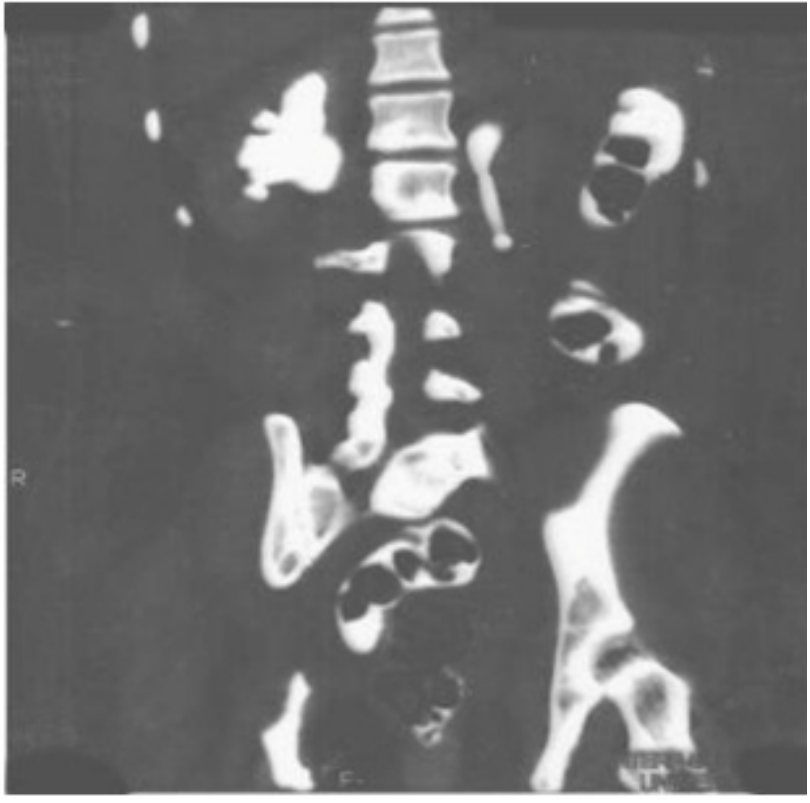

Figura 2 - TC de Abdome Superior (Técnica Multislice)

elevados (> 500U/L). A colestase intra-hepática da gravidez manifesta-se principalmente durante o terceiro trimestre, sendo o prurido o sintoma característico e a bilirrubina sérica raramente é superior a $6 \mathrm{mg} /$ dL. Colelitíase pode ocorrer a qualquer momento durante a gravidez, associada a dor no quadrante superior direito, febre e a ultrassonografia abdominal é utilizada para o diagnóstico. Todas estas manifestações foram descartadas no caso descrito, com base na 
história da paciente, aspectos clínicos e anormalidades bioquímicas. Porém, o diagnóstico de EHAG pode ser desafiador, pois a apresentação clínica pode ser inespecífica. ${ }^{13}$

As manifestações clínicas desse caso foram de EHAG no terceiro trimestre da gravidez, simulando outras hepatopatias com anormalidades funcionais do fígado, que com o diagnóstico precoce, compromete o prognóstico da gestante e do bebê. ${ }^{14,15}$ Diante disso, a estabilização clínica e hemodinâmica materna deve ser alcançada o mais rápido possível antes do parto, que inclui manter vias aéreas pérveas, tratamento para hipertensão, e correção de distúrbios de hipoglicemia, hidroeletrolíticos e de coagulação. Para isso, deve-se administrar cuidadosamente fluidos e hemoderivados endovenosos, bem como avaliar frequentemente sinais vitais e alterações do estado mental. ${ }^{16} \mathrm{O}$ parto vaginal é a melhor abordagem nessas gestantes, se tolerado; no entanto, cesariana é realizada frequentemente pelo risco de rápida deterioração do estado materno e fetal. No pós-parto, monitorização hemodinâmica é necessária, pois pacientes com EHAG possuem alto risco de hemorragias pela coagulopatia, além do risco de hipoglicemia. Não se deve negligenciar outras complicações que geralmente se desenvolvem após início da doença hepática, como pancreatite, disfunção renal e de vias urinárias.

Coagulopatia grave, icterícia, encefalopatia hepática, ascite, hipoglicemia e elevação de transaminases antes do parto (Tabela 1) são as principais características da EHAG. ${ }^{16-20}$ A biópsia de fígado é importante para o diagnóstico, mas nem sempre é possível especialmente em pacientes com coagulopatia grave. ${ }^{20}$ Tanto o ultra-som de abdome superior quanto a tomografia computadorizada abdominal podem demonstrar infiltração de gordura no fígado; no entanto, a sensibilidade e a especificidades destes estudos de imagem são insuficientes para fazer um diagnóstico definitivo de EHAG, sendo comuns os falsos negativos. ${ }^{20,21}$

O risco de progressão para IHA é elevado, entretanto a EHAG é considerada uma forma reversível de insuficiência hepática aguda com a interrupção da gravidez, podendo mesmo assim necessitar de transplante de fígado, devido insuficiência hepática persistente ou progressiva após o parto. Melhores resultados podem ser alcançados em centros multidisciplinares, onde existe uma possibilidade de uma equipe com ginecologistas/obstetras, hepatologistas, cirurgiões e anestesistas. Atualmente, a mortalidade materna diminuiu para menos de $10 \%$ pelo diagnóstico precoce da doença resultando em interrupção da gestação e cuidados intensivos durante o período pósparto.

A mortalidade materna é elevada em muitos casos, em lista de espera para transplante de fígado e o transplante de fígado raramente tem sido utilizado para EHAG. Levantamento estatístico recente americano da United Network for Organ Sharing (UNOS) revelou que durante em um período de 16 anos, entre

Tabela 1: Níveis séricos de aminotransferases (AST e ALT) na admissão hospitalar.

\begin{tabular}{lccc}
\hline Autor & Número de casos & Exame & Admissão \\
\hline Wei et al [16] & 12 casos & AST & $46-578$ \\
& & ALT & $67-425$ \\
McClements [17] & 03 casos & AST & $139-373$ \\
& & ALT & $108-435$ \\
Riyami et al. [18] & 01 Caso & AST & 118,9 \\
& & ALT & 129,8 \\
Vora et al [19] & & & 208 \\
& & AST & 304 \\
Remiszewski [20] & & ALT & 116 \\
& & AST & 137 \\
\hline
\end{tabular}

*Valores normais em mulheres: TGO 9-25 U/I, TGP 7-30 U/I. 
1987 e 2003, foram realizados apenas oito transplantes de fígado paraesta condição de gravidez. ${ }^{22,23}$ Portanto, é sugerido que o transplante hepático seja indicado para aquelas mulheres com insuficiência hepática fulminante, devido à EHAG, que manifestam sinais de insuficiência hepática irreversível.

Com o aumento do conhecimento e diagnóstico de EHAG, especialmente no reconhecimento precoce dos casos mais leves, e intervenção precoce e imediata, incluindo interrupção da gravidez por cesariana e infusão de plasma fresco congelado e albumina, alternadamente, o prognóstico pode ser melhor.

\section{Conflito de interesses}

Não há conflito de interesses.

\section{Referências}

1. Sheehan HL. The pathology of acute yellow atrophy and delayed chloroform poisoning. J Obstet Gynaecol. Br Emp. 1940;47:49-62.

2. Vigil-de Gracia P, Montufar-Rueda C. Acute fatty liver of pregnancy: diagnosis, treatment, and outcome based on 35 consecutive cases. J Matern Fetal Neonatal Med. 2011;24:11436.

3. Kaplan MM. Acute fatty liver of pregnancy. $\mathrm{N}$ Engl $\mathrm{J}$ Med. 1985;313:367-70.

4. Cunningham FG, Leveno KJ, Bloom SL, et al. Williams Obstetrics. 22nd ed. New York: McGraw-Hill; 2005.

5. Lee NM, Brady CW. Liver disease in pregnancy. World $J$ Gastroenterol. 2009;15:897-906.

6. Pinarbasi E, Percin FE, Yilmaz M, Akgun E, Cetin M, Cetin A. Association of microsomal epoxide hydrolase gene polymorphism and pre-eclampsia in Turkish women. J Obstet Gynaecol Res. 2007;33:32-7.

7. Steingrub JS. Pregnancy-associated severe liver dysfunction. Crit Care Clin. 2004;20:763-76.

8. Hepburn IS, Schade RR. Pregnancy-associated liver disorders. Dig Dis Sci. 2008;53: 2334-58.
9. Knox TA, Olans LB. Liver disease in pregnancy. N Engl J Med. 1996;335:569-76.

10. Ibdah JA. Acute fatty liver of pregnancy: an update on pathogenesis and clinical implications. World J Gastroenterol. 2006;12:7397-404.

11. Fesenmeier MF, Coppage KH, Lambers DS, Barton JR, Sibai BM. Acute fatty liver of pregnancy in 3 tertiary care centers. Am J Obstet Gynecol. 2005;192:1416-9.

12. Rajasri AG, Srestha R, Mitchell J. Acute fatty liver of pregnancy (AFLP)--an overview. J Obstet Gynaecol. 2007;27:23740.

13. Riely CA. Liver disease in the pregnant patient. Am J Gastroenterol. 1999;94:1728-32.

14. Rahman TM, Wendon J. Severe hepatic dysfunction in pregnancy. Q J Med. 2002;95:343-57.

15. Ko H, Yoshida EM. Acute fatty liver of pregnancy. Can J Gastroenterol. 2006;20:25-30.

16. Wei Q, Zhang L, Liu X. Clinical diagnosis and treatment of acute fatty liver of pregnancy: a literature review and 11 new cases. J Obstet Gynaecol Res. 2010;36:751-6.

17. McClements BM, Callender ME. Idiopathic acute fatty liver of pregnancy: three cases including a subsequent normal pregnancy. Ulster Med J. 1990;59:217-20.

18. Riyami NA, Al-Harthy A, Zia F. Atypical case of acute Fatty liver of pregnancy. Sultan Qaboos Univ Med J. 2011;11:50710.

19. Vora KS, Shah VR, Parikh GP. Acute fatty liver of pregnancy: a case report of an uncommon disease. Indian J Crit Care Med. 2009;13:34-6.

20. Remiszewski P, Pawlak J, Skwarek A, Grzelak I, Patkowski W, Grodzicki M, et al. Orthotopic liver transplantation for acute liver failure resulting from "acute fatty liver of pregnancy". Ann Transplant. 2003;8:8-11.

21. Usta IM, Barton JR, Amon EA, Gonzalez A, Sibai BM. Acute fatty liver of pregnancy: An experience in the diagnosis and management of fourteen cases. Am J Obstet Gynecol. 1994;171:1342-7.

22. Campillo B, Bernuau J, Witz MO, Lorphelin JM, Degott C, Rueff B, Benhamou JP. Ultrasonography in acute fatty liver of pregnancy. Ann Intern Med. 1986;105:383-4.

23. United Network for Organ Sharing. <www.unos.org > (Version current at September 8, 2005) 